\title{
Differential DNA copy number aberrations in the progression of cervical lesions to invasive cervical carcinoma
}

\author{
EUN KYEONG OH ${ }^{1}$, YONG-WAN KIM ${ }^{2}$, IN-WOOK KIM ${ }^{2}$, HAI-BO LIU ${ }^{2}$, KEUN-HO LEE $^{1}$, HEUNG JAE CHUN $^{3}$, \\ DONG CHOON PARK ${ }^{1}$, EUN-JEE OH ${ }^{4}$, AH WON LEE $^{4}, \mathrm{SU}_{\text {MI BAE }}^{2}$ and WOONG SHICK AHN ${ }^{1}$ \\ ${ }^{1}$ Department of Obstetrics and Gynecology, ${ }^{2}$ Catholic Research Institutes of Medical Science, \\ ${ }^{3}$ Institute of Cell and Tissue Engineering, ${ }^{4}$ Department of Pathology, College of Medicine, \\ The Catholic University of Korea, Seocho-ku, Seoul 137-040, Republic of Korea
}

Received April 25, 2012; Accepted June 21, 2012

DOI: $10.3892 /$ ijo.2012.1644

\begin{abstract}
Host genomic alterations in addition to human papillomavirus (HPV) are needed for cervical precursor lesions to progress to invasive cancer because only a small percentage of women infected by the virus develop disease. However, the genomic alterations during the progression of cervical lesions have not been systematically examined. The aim of this study was to identify differential genomic alterations among cervical intraepithelial neoplasia CIN1, CIN2, CIN3 and cervical squamous cell carcinoma (SCC). Genomic alterations were examined for 15 cases each of CIN1, CIN2, CIN3 and SCC by array-based comparative genomic hybridization (array $\mathrm{CGH}$ ). The chromosomal regions showing significant differential in DNA copy number aberrations (DCNAs) among CIN1, CIN2, CIN3 and SCC were successfully identified by resampling-based t-test. The chromosomal regions of 5q35.3 and 2q14.3 showed significant DCNAs between CIN1 and CIN2, and between CIN2 and CIN3, respectively, while a significant difference in DCNAs between CIN3 and SCC was observed at 1q24.3, 3p14.1, 3p14.2, 5q13.2, 7p15.3, 7q22.1 and $13 q 32.3$. In addition, the status of DCNAs in 1q43, 2 p11.2, 6p11.2, 7p21.1, 7p14.3, 10q24.1, 13q22.3, 13q34 and 16p13.3 was conserved throughout the progression of CIN to SCC. The presence of differential and common DCNAs among CIN1, CIN2, CIN3 and SCC supports that the CIN progression may include continual clonal selection and evolution. This approach also identified 34 probe sets consistently overexpressed when amplified, suggesting an unbiased identification of candidate genes in SCC during cervical cancer progression.
\end{abstract}

Correspondence to: Dr Woong Shick Ahn, Department of Obstetrics and Gynecology, College of Medicine, The Catholic University of Korea, 505 Banpodong, Seocho-ku, Seoul 137-040, Republic of Korea E-mail: ahnlab1@catholic.ac.kr

Key words: human papillomavirus, cervical intraepithelial neoplasia, squamous cell carcinoma, array-based comparative genomic hybridization, differential DNA copy number aberration

\section{Introduction}

Cervical cancer is the second most common malignancy in women worldwide. Approximately $80 \%$ of these tumors are squamous cell carcinomas (SCCs) and 5-20\% are adenocarcinomas (AdCAs) $(1,2)$. Cervical SCCs are often preceded by a premalignant disease known as cervical intraepithelial neoplasia (CIN) which is graded 1-3 with increasing atypia. Infection with high-risk subtypes of human papillomavirus (HPV), namely HPV-16 and HPV-18, is the major etiological factor and is the primary initiator of premalignant lesions (3). The viral oncoproteins E6 and E7 play an important role in the process of CIN, inhibiting a variety of cellular targets, including the tumor suppressor protein p53 and retinoblastoma (Rb), which disrupts key cellular processes such as apoptosis and cell cycle control processes and leads to genomic instability and neoplastic development. However, only a small fraction of women infected with oncogenic HPV subtypes develop cervical cancer, which indicates that HPV infection alone is not sufficient to cause disease and that there are other host factors associated with the development of invasive cervical cancer (4).

The process of increased genomic instability in addition to HPV infection has been proposed to contribute to the need for precursor lesions to progress to invasive cancer $(4,5)$. Progression from CIN1 to CIN3 and SCC is admitted and is consistent with the concept of lesional continuum $(6,7)$. However, because of the elevated rate of spontaneous regression of CIN1, it is probably a lesion of very low potential aggressivity and its role as a precursor is uncertain (8). Recently, It has been shown that genomic profiling of $16^{\mathrm{INK} 4 \mathrm{a}}$ immunopositive CIN2/3 lesions can distinguish histologically similar high-grade CIN lesions into potentially early and more advanced lesions (9). This indicates that the progression of CIN might be reflected in its chromosomal profile. In addition, the involvement of clonal selection and expansion process during the progression of CIN was also suggested (10).

However, the chromosomal aberrations which might affect the progression of cervical lesion to SCC have not been systematically examined. The differential and common chromosomal aberrations among CIN1, CIN2, CIN3 and SCC might be important clues for understanding of the progression of cervical lesions. In this study, we applied array-based comparative 
genomic hybridization (array $\mathrm{CGH}$ ) to specimens of 60 patients to identify differential and common chromosomal aberrations among CIN1, CIN2, CIN3 and cervical squamous cell carcinoma (SCC). Using an open database, our study showed driver genes to genetic alterations that provide tumor cells with a growth advantage during carcinogenesis or tumor progression.

\section{Materials and methods}

Tissue specimens. Sixty cervical tissue samples including 15 cases each of CIN1, CIN2, CIN3 and SCC were obtained from the Department of Obstetrics and Gynecology, Seoul St. Mary's Hospital, The Catholic University of Korea. This study followed the guidelines of the Institutional Review Board of The Catholic University of Korea and written informed consent was obtained from all patients included.

Microdissection, extraction of nucleic acids and HPV DNA testing. Paraffin-embedded tissues were first sectioned in $10 \mu \mathrm{m}$ slices, which were hematoxylin-eosin stained for selection of the appropriated tissue area. The corresponding selected areas of each tissue sample were then collected under microscopic observation with a 30-gauge needle (Becton-Dickinson, Franklin Lakes, NJ). Genomic DNA of microdissected tissue was extracted by proteinase $\mathrm{K}$ digestion followed by standard phenolchloroform extraction (11). HPV DNA test was performed with the hybrid capture II (HCII) assay system according to the manufacturer's instructions (Digene Diagnostics Inc., Gaithersburg, MD). Briefly, the isolated DNA from the above microdissected sample were denatured at $65^{\circ} \mathrm{C}$ for $45 \mathrm{~min}$ and hybridized under high stringency conditions with a mixture of RNA probes that detect 13 different high-risk HPV types: 16, 18, 31, 33, 35, 39, $45,51,52,56,58,59$ and 68 . The resultant DNA-RNA hybrids were captured on the surface of the microtiter plate wells coated with anti-DNA-RNA hybrid antibody. The immobilized hybrids were then reacted with an alkaline phosphatase-conjugated antihybrid monoclonal antibody. Light intensity was measured with a luminometer. A positive cutoff value was set at $1 \mathrm{pg}$ HPV DNA per $\mathrm{mm}$ in each specimen.

Array $C G H$. The array $\mathrm{CGH}$ experiments were performed with a MacArray ${ }^{\mathrm{TM}}$ Karyo 1440 (Macrogen, Seoul, Korea) according to the manufacturer's instructions (http://www. macrogen.co.kr/eng/biochip/karyo_summary.jsp) where the BAC chip information together with chromosomal location of each clone was also provided. The array consisted of 1,440 human BAC clones spotted in triplicate, including 356 cancerrelated genes at an average interval of $2.3 \mathrm{Mb}$. Sample DNA and 9948 male reference DNA (Promega Corp., Madison, WI) (500 ng each) were labeled by the random priming method with fluorescence dyes, $\mathrm{Cy} 3$ and $\mathrm{Cy} 5$, respectively. The use of a male reference DNA enabled the determination of whether the hybridization had succeeded based on the expected gain of chromosome $\mathrm{X}$ and loss of chromosome $\mathrm{Y}$ in the female test samples. The labeled DNAs were mixed with Cot-1 DNA (50 $\mu \mathrm{g}$; Gibco BRL, Gaithersburg, MD) and then hybridized to the array slides for 2 days at $37^{\circ} \mathrm{C}$ in a moist chamber. The array slides were rinsed in a washing buffer and dried well. The array slides were scanned using a GenePix 4000A scanner (Axon Instruments, Union City,
Table I. Patient characteristics according to cytopathology.

\begin{tabular}{lcccc}
\hline & CIN 1 & CIN2 & CIN3 & SCC \\
\hline No. of patients & 15 & 15 & 15 & 15 \\
Age (years) & & & & \\
Mean & $40.4 \pm 6.0$ & $37.3 \pm 7.6$ & $42.7 \pm 13.4$ & $53.0 \pm 11.3$ \\
HPV infection & & & & \\
Positive & 12 & 14 & 15 & 15 \\
Negative & 3 & 1 & 0 & 0 \\
\hline
\end{tabular}

CA). Spots were quantified using the MAC View ${ }^{\mathrm{TM}}$ software program (Macrogen) with the flagging of poor quality spots.

Array CGH data analysis. After exclusion of clones with one or more flagged spots, the average of the triplicate spots was calculated for each $\mathrm{BAC}$ clone. $\log _{2} \mathrm{Cy} 3 / \mathrm{Cy} 5$ ratios were normalized using the locally weighted regression known as lowess smoothing for each array followed by scale normalization between arrays with R package limma (www.r-project. org). Selection of abnormal clones used the averaged $\log _{2}$ ratio for each type of cervical lesion. Chromosomal aberrations were classified as a gain when the normalized $\log _{2}$ ratio was $\geq 0.2$ and as a loss when the ratio was $\leq-0.2$. This number was determined as a 3-fold difference of the average standard deviation of normal versus normal array $\mathrm{CGH}$ hybridization. In addition, the $\log _{2}$ ratio $>0.6$ was defined as amplification. After selection of clones with aberrations, resampling-based t-test and multiple testing with R package multi-test (www.r-project.org) were performed to identify BAC clones showing significantly differential DNA copy number aberration (DCNA) between and among CIN1, CIN2, CIN3 and SCC, respectively. The adjusted P-values based on permutation method (1000 permutations) $<0.05$ were considered statistically significant (12).

Validation of DCNA. Polymerase chain reaction (PCR) was carried out to test the reliability of chromosomal aberrations estimated by the array CGH experiments (13). PCR was conducted against pooled genomic DNA of 15 cases each of CIN1, CIN2, CIN3 and SCC rather than individual case because the average $\log _{2}$ ratio for each cervical lesion was used for the discrimination of DCNA. Two genes were selected including MTR and HDAC9, which show the same state of DCNA at CIN1, CIN2, CIN3 and SCC. PCR was performed in a reaction tube containing a PCR mixture composed of $1 \mathrm{ng}$ sample DNA, $20 \mu \mathrm{M}$ deoxynucleotide triphosphate (a mixture of dATP, dCTP, dGTP and dTTP), $2 \mu \mathrm{l}$ 10X PCR buffer (50 mM KCl, $4 \mathrm{mM} \mathrm{MgCl}_{2}, 10 \mathrm{mM}$ Tris- $\mathrm{HCl}$, $\mathrm{pH} 8.3$ ), $20 \mathrm{pM}$ of each primer, and 2.5U Taq polymerase (Takara, Shiga, Japan). The 9947A female reference DNA was obtained from Promega and $\beta$-globin was used as internal control. The mixture was cycled in a PTC-200 thermal cycler (MJ Research, Waltham, MA) at $94^{\circ} \mathrm{C}$ for $30 \mathrm{sec}, 48^{\circ} \mathrm{C}$ for $30 \mathrm{sec}$ and $72^{\circ} \mathrm{C}$ for $30 \mathrm{sec}$ for 35 cycles. PCR products were analyzed by electrophoresis on an agarose gel. The amplicons were visualized with an ultraviolet transilluminator Chemi Imager ${ }^{\mathrm{TM}} 4400$ (Alpha Innotech, San Leandro, CA). 


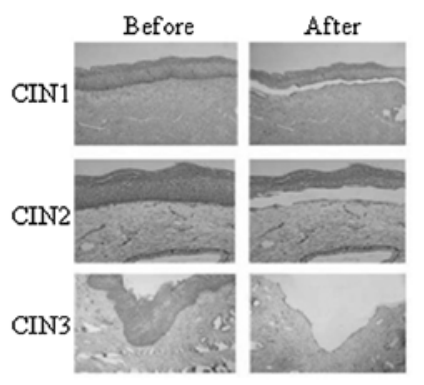

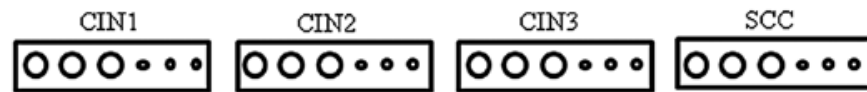

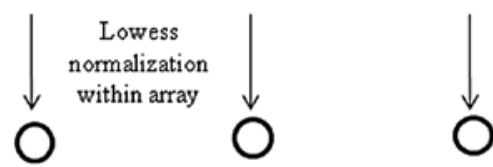

$\log _{2}$ ratio $\geq 0.2$ or $\log _{2}$ ratio $\leq-0.2$

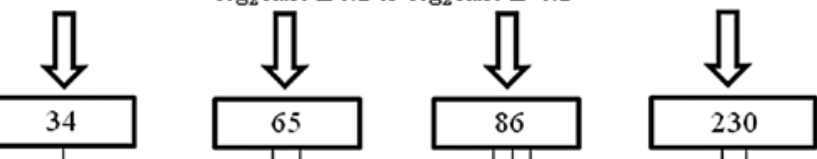

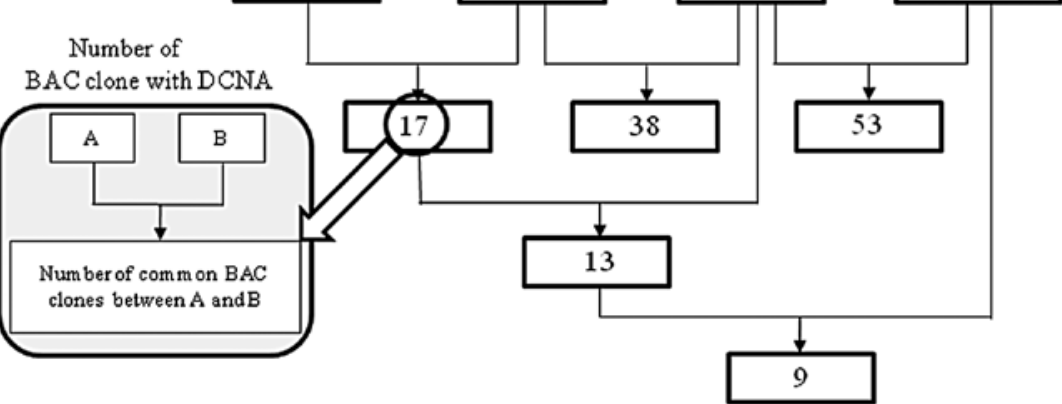

Figure 1. Schematic diagram for the identification of BAC clones with DCNA from array CGH data. (a) Images of cervical intraepithelial neoplasia before (left) and after (right) microdissection by 30-gauge needle (hematoxylin and eosin staining: original magnification x100). (b) Outline of analysis procedures with each sample showing the general steps required to identify DCNA that modulate a specific phenotype.
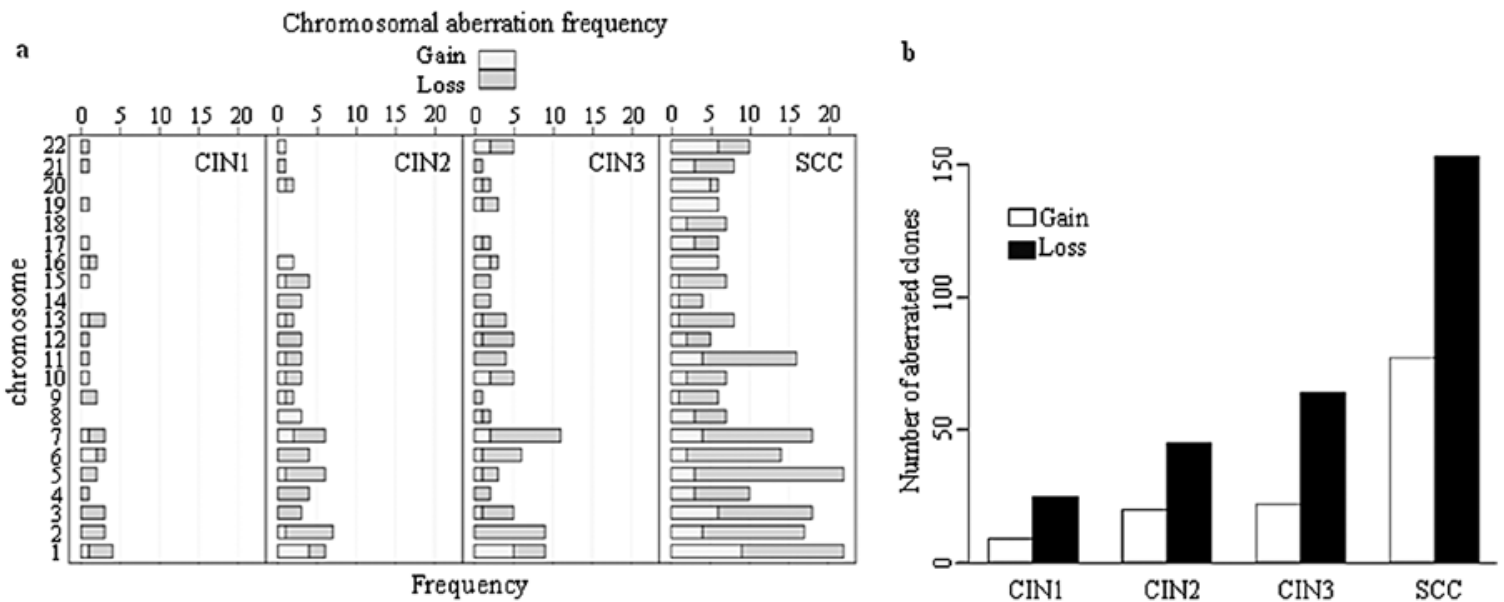

Figure 2. Distribution of BAC clones with DCNA along chromosomes. (a) Gained and lost regions in the study population. (b) Number of chromosomal aberrations among CIN1, CIN2, CIN3, and SCC samples.

Cervical squamous cell carcinoma datasets in public database. To validate the data generated by array $\mathrm{CGH}$, we directly accessed another independent public cervical cancer gene expression datasets (GEO accession no. GSE7803) (14). In total, 38 microdissected squamous epithelial samples from 10 normal cervices, 7 high-grade squamous intra-epithelial lesions (HSIL) and 21 SCCs were profiled for differential gene expression discovery.

\section{Results}

Chromosomal aberrations in CIN1, CIN2, CIN3 and SCC. The mean age and HPV status of patients are shown in Table I. Ninety-three percent of patients showed infection with high-risk
HPV types and the mean ages of women were 40.4, 37.3, 42.7 and 53.0, respectively. We have microdissected 60 samples under a microscope to obtain DNA samples (Fig. 1a). To determine BAC clones with DCNA, the $\log _{2}$ ratios of 15 samples for each case were averaged (Fig. 1b). The sex chromosome and BAC clones with $>2$ missing values in each type were excluded from the analysis. A total of $276 \mathrm{BAC}$ clones showed at least a DCNA among CIN1, CIN2, CIN3 and SCC and the number of BAC clones with DCNA increased with the progression of CIN. Approximately half of the BAC clones with DCNA were also retained at the next grade of cervical lesion. This indicates that the clonal evolution and selection process might be involved in progression of CIN. Distribution of chromosomal aberration at each type of cervical lesion and SCC is shown in Fig. 2. The 
Table II. BAC clones with the same status of DCNA for CIN1, CIN2, CIN3 and SCC.

\begin{tabular}{|c|c|c|c|c|c|c|}
\hline \multirow[b]{2}{*}{$\begin{array}{l}\text { Clone } \\
\text { ID }\end{array}$} & \multirow[b]{2}{*}{$\begin{array}{c}\text { Chromosomal } \\
\text { region }\end{array}$} & \multirow[b]{2}{*}{ Candidate gene } & \multicolumn{4}{|c|}{ Copy no. aberration } \\
\hline & & & CIN1 & CIN2 & CIN3 & SCC \\
\hline 1157 & $1 q 43$ & MTR & - & - & - & - \\
\hline 408 & $2 \mathrm{p} 11.2$ & RPIA & - & - & - & - \\
\hline 998 & $6 \mathrm{p} 11.2$ & RAB23, PRIM2A & - & - & - & - \\
\hline 207 & $7 \mathrm{p} 21.1$ & HDAC9 & + & + & + & + \\
\hline 1329 & $7 \mathrm{p} 14.3$ & PDEIC & - & - & - & - \\
\hline 335 & $10 \mathrm{q} 24.1$ & KIAA0690, PGAM1, EXOSC1, ZDHHC16 & + & + & + & + \\
\hline 472 & $13 q 22.3$ & $E D N R B$ & - & - & - & - \\
\hline 709 & $13 q 34$ & GRTP1, DKFZp451A211 & + & + & + & + \\
\hline 456 & $16 \mathrm{p} 13.3$ & SOLH, LOC 146325, FLJ36208, PIGQ, RAB40C & + & + & + & ++ \\
\hline
\end{tabular}

-, loss (average $\log _{2}$ ratio $\leq-0.2$ ); + gain (average $\log _{2}$ ratio $\geq 0.2$ ); ++, amplification (average $\log _{2}$ ratio $>0.6$ ).
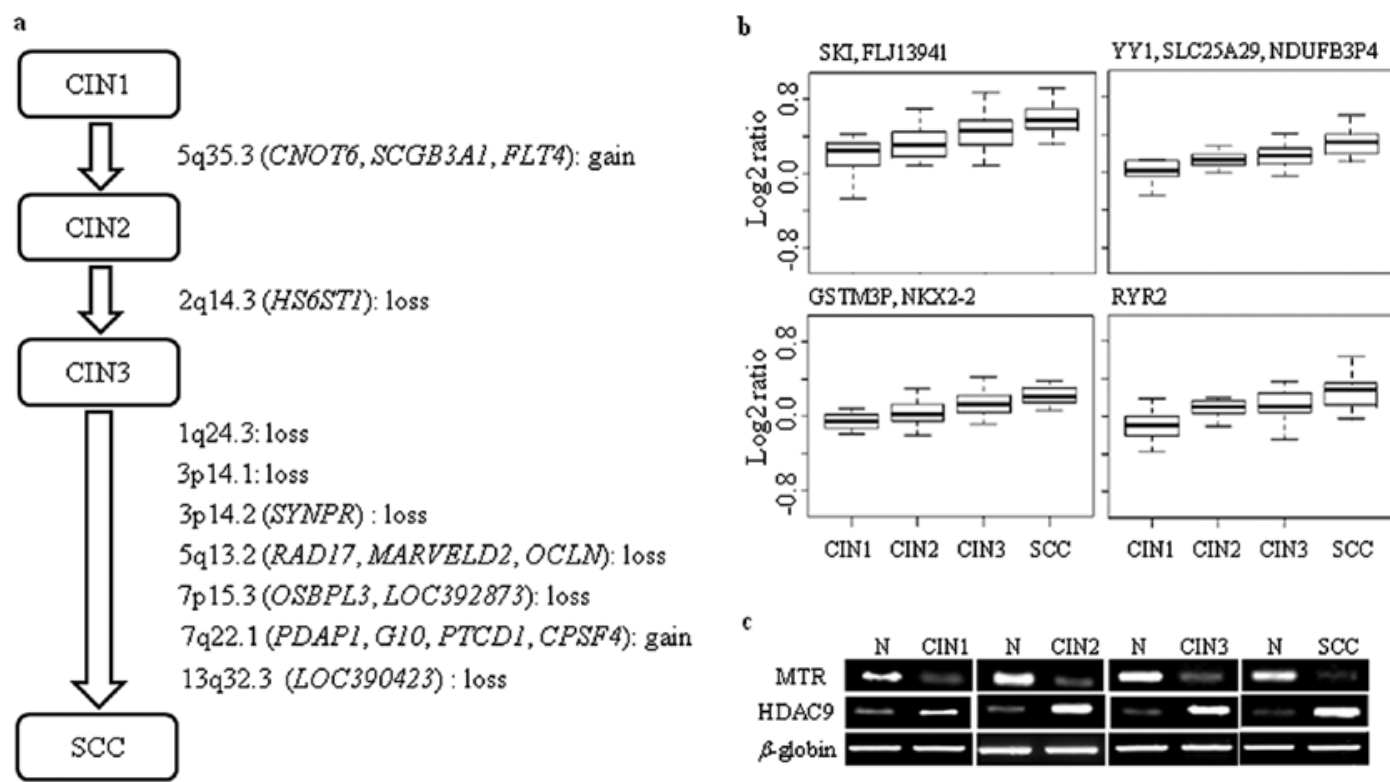

Figure 3. Differential DCNA during progression of CIN. (a) The BAC clones showing significant differential in DCNA during progression of CIN. (b) Box plot for copy number aberration distribution of specific genes. The $y$-axis reports the $\log _{2}$ value ratios. The box plots indicate the $\log _{2}$ value ratio with median value (small square). The solid lines within each box indicate the median. The rectangle indicates the interquartile range (IQR). (c) DCNA validation of MTR and HDAC9 in CIN1, CIN2, CIN3 and SCC by PCR. The loss of MTR and gain of HDAC9 is maintained during the progression of CIN to SCC.

number of chromosomes having $>5$ BAC clones with DCNA was markedly increased in SCC, which indicates that severe chromosomal instability might be required during transition from CIN3 to SCC.

The BAC clones showing the same status of DCNA at CIN1, CIN2, CIN3 and SCC were shown in Table II. These BAC clones included genes such as 5-methyltetrahydrofolate-homocysteine methyltransferase (MTR; loss), histone deacetylase (HDAC9; gain), calcium/calmodulin-dependent 3',5'-cyclic nucleotide phosphodiesterase (PDE1C; loss), component of the exosome $3^{\prime} \rightarrow 5^{\prime}$ exoribonuclease complex (EXOSC1; gain), zinc finger DHHC domain-containing protein 16 (ZDHHC16; gain), G protein-coupled receptor (EDNRB; loss), ethanolamine phosphate transferase (PIGO; gain), and
Ras-related protein (RAB40C; gain). The DCNAs of these nine $\mathrm{BAC}$ clones might have important role in the progress of cervical lesions because they were maintained throughout CIN progression to SCC.

Identification of BAC clones with significant differential in DCNA among CIN1, CIN2, CIN3 and SCC. The resamplingbased t-test and multiple testing were performed to detect BAC clones with significantly differential DCNA between and among CIN1, CIN2, CIN3 and SCC, respectively. The chromosomal regions of 5q35.3 and 2q14.3 showed significantly differential DCNA between CIN1 and CIN2, and between CIN2 and CIN3, respectively (Fig. 3a). The chromosomal region of 5q35.3 included poly(A) nuclease (CNOT6), potential growth inhibitory 
a

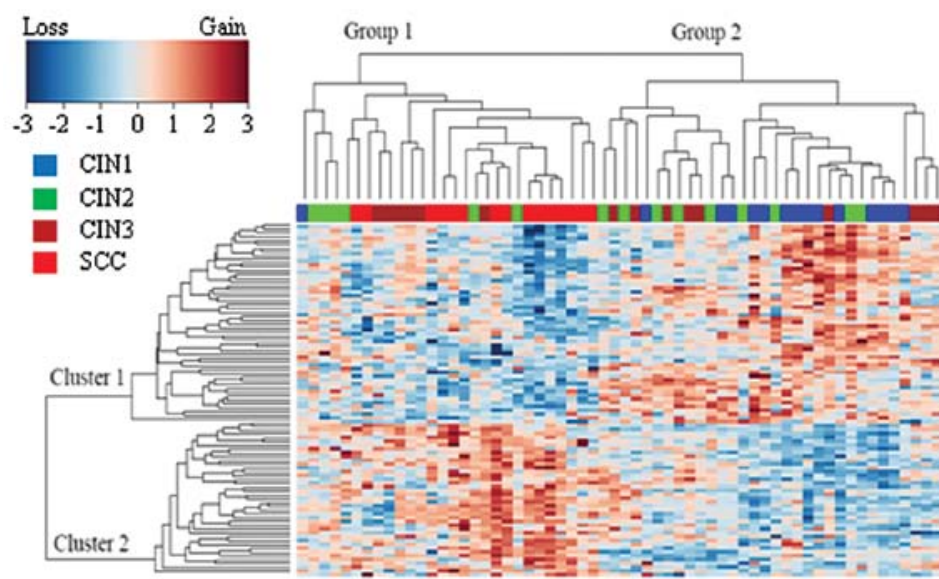

c

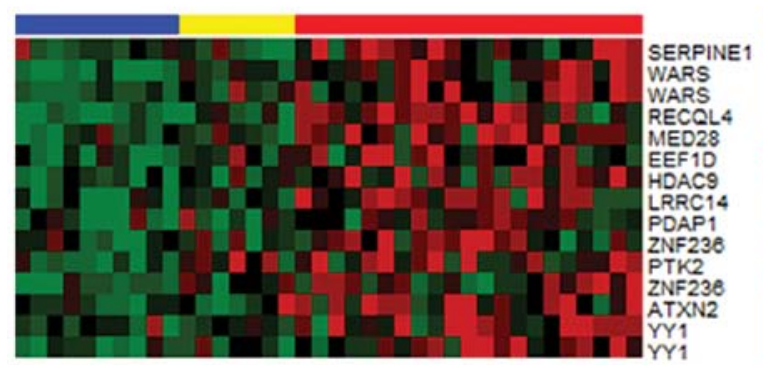

-3.3 Logratio 3.4

Normal HSIL $\square$ SCC

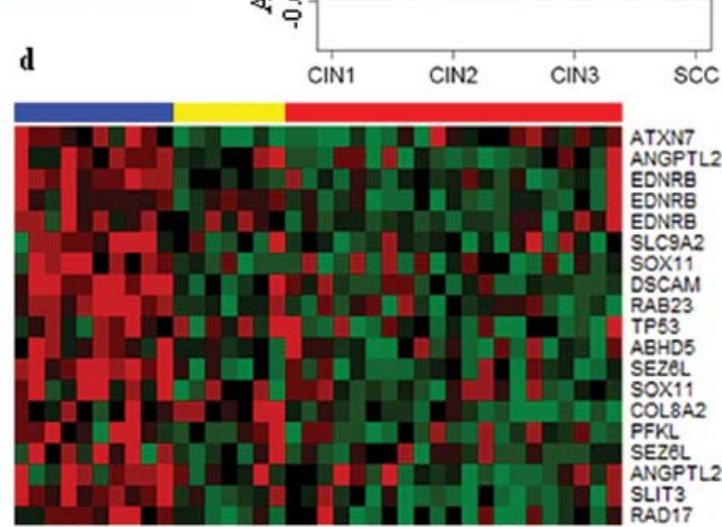

Figure 4. Two-way hierarchical clustering of 60 arrays each including 15 cases of CIN1, CIN2, CIN3 and SCC. (a) Clustering of 60 samples. Vertical columns correspond to samples, horizontal lines correspond to BAC clones. Red squares correspond to loss, green squares to gain, and grey squares are non-informative. (b) The average $\log _{2}$ ratio profiles of BAC clones which belong to clusters 1 and 2. Except for few BAC clones, the DNA copy number of BAC clones in cluster 1 tends to decrease while that in cluster 2 tends to increase with the progression of cervical lesion. (c) This analysis identified gene sets whose expression most strongly correlated with gain copy number in SCC as shown in Table III $(\mathrm{P}<0.05)$. Then, we performed a supervised clustering with the sets and samples such as normal, HSIL, and SCC. The genes are presented in matrix format, where rows represent individual genes and columns represent each tissue. Samples are ordered according to progression status of the tumors (blue, normal; yellow, HSIL; red, SCC). Each cell in the matrix represents the expression level of a gene in an individual tissue. Red and green cells reflect high and low expression levels, respectively. (d) This analysis identified gene sets whose expression most strongly correlated with loss copy number in SCC $(\mathrm{P}<0.05)$.

cytokine (SCGB3AI), and tyrosine kinase receptor for vascular endothelial growth factors C and D (FLT4). The loss of heparan sulfate biosynthetic enzyme (HS6ST1) located at 2q14.3 during the progression of CIN2 to CIN3 might lead to failure of heparan sulfate synthesis. In the case of progression of CIN3 to SCC, seven BAC clones showed significantly different DCNA. Both PDAPl and RADI7 showed DCNA only at SCC. This suggests that the gain of $P D A P 1$ and loss of RAD17 might be potential markers distinguishing SCC from CINs.

The multiple testing among CIN1, CIN2, CIN3 and SCC identified 57 BAC clones showing significant DCNA (Table III). Among them, 45 BAC clones showed DCNA only at SCC (Fig. 3b). This indicates that the marked increase of DCNA might be required for progression from CIN3 to SCC. The gains of 1p36.33-1p36.32 including SKI (v-ski sarcoma viral oncogene homolog), 5q35.3 including FLT4 and 8q24.3 including $\mathrm{ZC} 3 \mathrm{HDC} 3$ (zinc finger $\mathrm{CCCH}$ domain-containing protein 3) were maintained from CIN2 to SCC while the loss of 2q12.1 including SLC9A2 (sodium/hydrogen exchanger) was kept from CIN2 to SCC. These conserved DCNAs from CIN2 to SCC might play an important role in the progression of cervical lesions like the BAC clones in Table II. To verify the chromosomal aberrations estimated by array-CGH experiment, PCR was conducted against pooled genomic DNA of 15 cases each of CIN1, CIN2, CIN3 and SCC. The targets of PCR were two genes, the MTR and HDAC9, which showed the same state of DCNA at CIN1, CIN2, CIN3 and SCC (Table II). The PCR amplicons of MTR and HDAC 9 generated from test samples presented a clear contrast with those generated from normal samples, while there were no significant differences in the amplicons of $\beta$-globin between test and normal samples (Fig. 3c). This result showed a good agreement with DCNAs evaluated by array-CGH experiments.

Clustering of array-CGH data including CIN1, CIN2, CIN3 and SCC. To test the differentiability of chromosomal profile during CIN progression, hierarchical clustering was carried out for the $57 \mathrm{BAC}$ clones which showed significantly differential in DCNA among CIN1, CIN2, CIN3 and SCC (Table III). The clustering was done on both samples and BAC clones (Fig. 4a). Group 1 included 3\% CIN1, 19\% CIN2, 22\% CIN3 and 56\% SCC, while group 2 was composed of $43 \%$ CIN1, 30\% CIN2 and $27 \%$ CIN3. This suggests that CIN1 could be efficiently distinguished from SCC, while classification between CIN2 and 
Table III. Fifty-seven BAC clones with significantly different DCNA among CIN1, CIN2, CIN3 and SCC.

\begin{tabular}{|c|c|c|c|c|c|c|}
\hline \multirow[b]{2}{*}{$\begin{array}{l}\text { Clone } \\
\text { ID }\end{array}$} & \multirow[b]{2}{*}{$\begin{array}{c}\text { Chromosomal } \\
\text { region }\end{array}$} & \multirow[b]{2}{*}{ Candidate gene } & \multicolumn{4}{|c|}{ Copy no. aberration } \\
\hline & & & CIN1 & CIN2 & CIN3 & $\mathrm{SCC}$ \\
\hline 99 & $1 \mathrm{p} 34.3$ & COL8A2, CSF3R, RSPO1 & & & & - \\
\hline 1243 & $1 \mathrm{p} 33$ & LOC 388630 & & & & - \\
\hline 841 & $1 \mathrm{p} 31.3$ & KIAA1573 & & & & - \\
\hline 196 & $1 \mathrm{p} 13.2$ & $M R P 63 P 1$ & & & & - \\
\hline 1002 & 1q24.3 & MYOC, TNFSF6 & & - & & - \\
\hline 894 & $2 \mathrm{p} 25.2$ & SOX11, CMPK2 & & & & - \\
\hline 842 & $2 \mathrm{p} 14$ & FLJ16124 & & - & & - \\
\hline 857 & $2 \mathrm{q} 12.1$ & SLC9A2, MGC11332 & & - & - & - \\
\hline 950 & $3 \mathrm{p} 21.33$ & ABHD5 & & & & - \\
\hline 1005 & $3 \mathrm{p} 14.1$ & ATXN7, EPM5 & & & & - \\
\hline 1227 & $4 \mathrm{p} 15.33$ & BAPX1, LOC285548, FAM44A & & & & - \\
\hline 1009 & $4 \mathrm{p} 15.32$ & MED28, KIAA1276 & & & & - \\
\hline 816 & $4 \mathrm{q} 31.3$ & KIAA0922 & & - & & - \\
\hline 820 & $5 q 13.2$ & RAD17, MARVELD2, OCLN & & & & - \\
\hline 295 & $5 q 34-5 q 35.1$ & SLIT3 & & & & - \\
\hline 1093 & $5 q 35.1$ & KCNIP1 & & & & - \\
\hline 1133 & $6 \mathrm{p} 21.1$ & TRERF1 & & & & - \\
\hline 838 & $6 \mathrm{q} 24.3$ & LOC389432 & & & & - \\
\hline 168 & $6 \mathrm{q} 25.2$ & MYCTI, VIP & & & & - \\
\hline 1215 & $7 \mathrm{p} 15.3$ & OSBPL3, LOC392873 & & & & - \\
\hline 886 & $7 \mathrm{p} 15.2$ & KIAA0087, LOC442614 & & & & - \\
\hline 489 & $7 \mathrm{p} 13$ & LOC442745, NUDCD3, LOC442301 & & & - & - \\
\hline 222 & $7 \mathrm{q} 32.3$ & FLJ40288 & & & & - \\
\hline 318 & $8 \mathrm{q} 24.22$ & $T G, N D R G 1$ & & & & - \\
\hline 978 & $9 \mathrm{q} 33.3$ & ANGPTL2 & & & & - \\
\hline 1282 & $11 \mathrm{q} 23.3$ & APOA5, APOA1 & & & & - \\
\hline 1000 & $11 \mathrm{q} 24.1$ & $S C N 3 B$ & & & & - \\
\hline 1018 & $13 q 12.11$ & FEOM4, GJA3 & & & & - \\
\hline 1006 & $13 \mathrm{q} 32.3$ & LOC390423 & & & & - \\
\hline 4 & $17 \mathrm{p} 13.1$ & FXR2, SAT2, SHBG, ATP1B2, TP53 & & & & - \\
\hline 189 & $17 \mathrm{q} 11.2$ & HCA66, SUZ12, LOC114659 & & & & - \\
\hline 1098 & $21 \mathrm{q} 22.2$ & C21orf24, ETS2, FLJ45139 & & & & - \\
\hline 345 & $21 \mathrm{q} 22.2$ & DSCAM & & & & - \\
\hline 828 & 22q12.1 & SEZ6L, LOC57168, HPS4 & & & & - \\
\hline 460 & 22q12.1 & KIAA1043 & & & & - \\
\hline 1391 & $1 \mathrm{p} 36.33$ & PRKCZ, FLJ31031, LOC440554, SKI & & + & + & + \\
\hline 122 & 1p36.33-1p36.32 & SKI, FLJ13941 & & + & + & + \\
\hline 1594 & $1 q 43$ & RYR2 & & & & + \\
\hline 1534 & $2 q 35$ & WNT6, WNT10A, LOC391485 & & & & + \\
\hline 1562 & $3 q 26.31$ & GHSR, SPATA16 & & & + & + \\
\hline 1167 & $3 q 27.3$ & $S S T$ & & & & + \\
\hline 1339 & 4p16.1 & SLC2A9 & & & & + \\
\hline 1387 & $5 q 31.3$ & 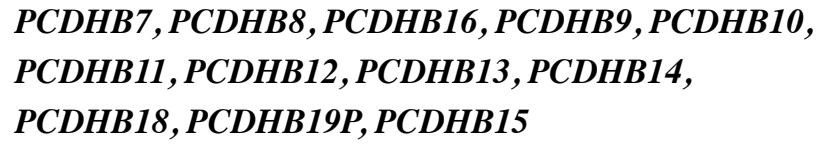 & & & & + \\
\hline
\end{tabular}


Table III. Continued.

\begin{tabular}{|c|c|c|c|c|c|c|}
\hline \multirow[b]{2}{*}{$\begin{array}{l}\text { Clone } \\
\text { ID }\end{array}$} & \multirow[b]{2}{*}{$\begin{array}{l}\text { Chromosomal } \\
\text { region }\end{array}$} & \multirow[b]{2}{*}{ Candidate gene } & \multicolumn{4}{|c|}{ Copy no. aberration } \\
\hline & & & CIN1 & CIN2 & CIN3 & SCC \\
\hline 174 & $5 q 35.3$ & CNOT6, SCGB3A1, FLT4 & & + & + & + \\
\hline 1272 & $7 q 22.1$ & PDAP1, G10, PTCD1, CPSF4, ATP5J2 & & & & + \\
\hline 1580 & $7 q 22.1$ & $\begin{array}{l}\text { SERPINE1, AP1S1, VGF, FLJ39237, MOGAT3, PLOD3, } \\
\text { ZNHIT1, CLDN15, TTC11 }\end{array}$ & & & & + \\
\hline 1284 & $8 q 24.3$ & ZC3HDC3, GSDMDC1, PP3856, EEF 1D, PTK2 & & + & + & + \\
\hline 607 & $8 q 24.3$ & $\begin{array}{l}\text { CYHR1, KIFC2, FOXH1, PPP1R16A, GPT, LOC113655, } \\
\text { RECQL4, LRRC14, LOC441381, MGC70857, KIAA1688 }\end{array}$ & & + & & + \\
\hline 1203 & $11 \mathrm{p} 15.5$ & TOLLIP, BRSK2 & & & & + \\
\hline 1389 & $12 q 24.12$ & $\operatorname{ATXN2}$ & & & + & + \\
\hline 363 & $14 q 32.2$ & YY1, SLC25A29, C14orf68, WARS, NDUFB3P4 & & & & + \\
\hline 760 & $16 p 11.2$ & SBK1,LOC388237, LOC388229 & & & & + \\
\hline 1083 & $18 p 11.22$ & C18orf30, C18orf58 & & & & + \\
\hline 312 & $18 q 23$ & ZNF236, MBP & & & & + \\
\hline 1589 & 20p13 & ATRN, GFRA4, ADAM33 & & & & + \\
\hline 1581 & $20 \mathrm{p} 11.22$ & GSTM3P, NKX2-2 & & & & + \\
\hline 260 & $21 q 22.3$ & AIRE, PFKL, C21orf 2, TRPM2 & & & & + \\
\hline
\end{tabular}

- , loss (average $\log _{2}$ ratio $\leq-0.2$ ); + , gain (average $\log _{2}$ ratio $\geq 0.2$ ). The gain BAC clones at SCC are marked in bold.

CIN3 might be difficult. The CIN2/3s in group 1 including all cases of SCC might have a high potential to progress to SCC, while the CIN2/3s of group 2 including $93 \%$ of CIN1s might have a high potential to regress or persist. Groups 1 and 2 seemed to have contrast pattern between cluster 1 and 2 . This was more apparently observed when the average $\log _{2}$ ratios were plotted against each type of cervical lesion (Fig. $4 \mathrm{~b}$ ). The BAC clones which belong to cluster 1 and 2 showed contrast pattern in terms of the average $\log _{2}$ ratio. CIN2 and CIN3 appeared to be a kind of intermediate state, where only minor change was evident, with a few exceptions. This indicates that severe chromosomal aberrations might not occur during the progression of CIN2 to CIN3. To validate the genes located in a region of genomic copy gain/loss in SCC, we directly accessed another independent public cervical cancer gene expression datasets (GEO accession no. GSE7803) (14). Differentially expressed genes among the genes in Table III were identified by comparison of SCCs to normal cervix samples $(\mathrm{P}<0.05)$ from a one-way ANOVA. This comparison yielded 15 probe sets with higher expression in SCCs and 19 genes with lower expression in SCCs. A heat map of expression for 15 genes (SERPINE1, WARS, RECQL4, MED28, EEF1D, HDAC9, LRRC14, PDAP1, ZNF236, PTK2, ATXN2, and YY1) that correlated with gain copy number in SCC is shown in Fig. 4c. This analysis also showed 19 probe sets (ATXN7, ANGPTL2, EDNRB, SLC9A2, SOX11, DSCAM, RAB23, TP53, ABHD5, SEZ6L, COL8A2, PFKL, SEZ6L, SLIT3, and RAD17) that were correlated with loss copy number in SCC (Fig. 4d). Using 34 probesets as a signature, the SCCs showed a dominant feature of expression pattern of the gene sets compared to HSILs and normal cervix samples. The results suggest that the 34 probes might correlate with CIN progression to invasive cervical carcinoma.

\section{Discussion}

Integration of high-risk HPV associated with host genomic alterations has been provided in a study that showed more numerical chromosomal aberrations were found to progress to invasive cancer for precursor lesions (15). The present study surveyed the chromosomal aberrations for CIN1, CIN2, CIN3 and SCC with array-CGH to determine whether the progression of CIN is reflected in their genomic signature. The DCNAs of chromosomal locations including 1q43, 2p11.2, 6p11.2, 7p21.1, 7p14.3, 10q24.1, 13q22.3, 13q34 and $16 \mathrm{p} 13.3$ might play a crucial role in the progression of cervical lesion because they were conserved throughout the progression of CIN to SCC. The chromosomal region of 16p13.3 showed amplification at SCC (16). HDAC9 located in $7 \mathrm{p} 21.1$ is involved in the alteration of chromosome structure and affects the transcription factor access to DNA. HDAC9 are significantly upregulated in high-risk medulloblastoma in comparison to low-risk medulloblastoma, and their expression is associated with poor survival (17). The amplification of $R A S$ oncogene family, $R A B 40 C$, might be a potential maker for SCC (18). The DCNAs of these nine BAC clones might have important role in the progress of cervical lesions 
because they were maintained throughout CIN progression to SCC.

The chromosomal regions of 5q35.3 and 2q14.3 showed significant differential in DCNA between CIN1 and CIN2, and between CIN2 and CIN3, respectively. The gain of 5q35.3 including FLT4 at CIN2 might induce the activation of lymphangiogenesis and maintenance of the lymphatic endothelium, while the loss of 2q14.3 including HS6ST1 at CIN3 might lead to failure in generating a myriad of distinct heparan sulfate fine structures that carry out multiple biological activities. The gain of FLT4 might play important role in the progression of CIN1 to CIN2 because the vascular endothelial growth factor (VEGF) C/FLT4 autocrine loop in tumor cells is known as a potential enhancer system to promote cancer progression (19). The heparan sulfate biosynthetic enzyme family is key components in generating a myriad of distinct heparan sulfate fine structures that carry out multiple biological activities (20). The chromosomal regions with significantly differential DCNA were markedly increased in the progression of CIN3 to SCC and 55\% of newly appeared clones of DCNA at SCC included at least a cancer associated gene. Region on chromosome 7q22 was commonly gained, whereas chromosome $5 \mathrm{q}$ frequently showed losses in cervical malignant lesions $(21,22)$. Among genes included in those seven BAC clones, PDAP1 located at 7q22.1 is known to enhance plateletderived growth factor A (PDGFA)-stimulated cell growth in fibroblasts, but inhibits the mitogenic effect of PDGFB while $R A D 17$ located at $5 \mathrm{q} 13.2$ encodes a product that is highly similar to the gene product of $S$. pombe rad17, a cell cycle checkpoint gene required for cell cycle arrest and DNA damage repair in response to DNA damage (23). In addition, both $P D A P 1$ and $R A D 17$ showed DCNA only at SCC. This implicates that the SCC could have different chromosome profile from CINs (24). As it is difficult to distinguish between CIN3 lesion and early invasive SCC on clinical grounds alone, the gain of PDAPI and loss of $R A D 17$ might be potential markers distinguishing SCC from CINs (25).

The multiple testing among CIN1, CIN2, CIN3 and SCC identified 57 BAC clones showing significant DCNA. The marked increase of DCNA at SCC only might be required for progression from CIN3 to SCC. The gains of 1p36.33 including SKI and 5q35.3 including FLT4 were maintained from CIN2 to $\mathrm{SCC}$, which were not common chromosomal aberrations. There have been many reports on chromosomal aberrations, including duplication or triplication and deletions in $1 \mathrm{p} 36.33$ and $5 q 35.3$, associated with various genetic diseases $(26,27)$. It is however likely that deletion is more common in both aberrations than either duplication or triplication (28). The $8 \mathrm{q} 24$ amplicon has been attributed to PTK2 (which encodes $F A K$ ) in squamous carcinoma cell lines (29). PTK2/FAK encodes a cytoplasmic tyrosine kinase, and seems to be specific to the ability of integrins to crosstalk through Ras and PI3K for oncogenesis (30). Integrin signaling seems to maintain the cancer stem cell population in tumors, as ablation of $P T K 2$ decreased the pool of cancer stem cells in spontaneously forming mouse mammary tumors (31). Thus the 8q24 amplicon has a plausible role in cancer biology. It is also consistent that the loss of 2q12.1 including SLC9A2 (sodium/hydrogen exchanger) was kept from CIN2 to SCC (21). These conserved DCNAs from CIN2 to SCC might play an important role in the progression of cervical lesions.
Seventy-nine percent of these BAC clones presented DCNA only at SCC. This indicates that the progression to SCC accompanies a dramatic increase in DCNA. The gain of oncogenes such as $R A B 40 C$ and $S K I$ was retained from CIN1 and CIN2, respectively, to SCC. The loss of tight junction membrane proteins such as MARVELD2 and OCLN at SCC might induce destruction of the epithelial barriers $(32,33)$. The severe DCNAs at SCC might be due to the genome instability by the integration of HPV DNA. The HPV initially exists in an episomal state after infection (34). However, the viral DNA often integrates into the host genome as lesions progress (35). The fact that most invasive cases contain integrated copies of the virus genome suggests that this event may be selected during clonal evolution and, thus, contributes to tumor development (36). The viral DNA integration into host genome leads to an increase of E6 and E7 transcription at the interruption of the viral E2 regulatory component, escalating the ability of the virus to induce neoplastic transformation (37). The viral integration may also be located within fragile sites or area of the genome that contain tumor suppressor genes or oncogenes $(38,39)$. In addition, DCNAs and other complex rearrangements have been observed near sites of viral integration (39). These reports implicate that HPV mediated tumorigenesis might act at the direct integrationrelated disruption of genomic segmental DNA copy number as well as at viral oncoproteins.

Gene expression is useful for identifying target genes within a region of genomic copy gain/loss. Gene expression data with DCNA could derive correlation of genes in determining the ranking of genes. Not all genes studied here, however, had a change in expression level. It was found that $62 \%$ of highly amplified genes in breast cancer exhibit $\geq 2$-fold increased expression (40). Another study showed that $44 \%$ of the highly amplified genes were overexpressed and $10.5 \%$ of the highly overexpressed genes were amplified in breast cancer cell lines (41). Overall, $\geq 12 \%$ of all variation in gene expression was directly attributed to variation in gene copy number. Transcription regulation, such as upstream genes and feedback regulators, is related to this unparalleled changes in the expression level. Our study identified 34 probesets consistently overexpressed when amplified, suggesting an unbiased identification of candidate drivers in SCC beyond transcription factors or signaling proteins.

In conclusion, the presence of differential and common DCNAs among CIN1, CIN2, CIN3 and SCC supports that the CIN progression might include continual clonal selection and evolution. In addition, the functional study of genes showing those DCNAs might give clues to understanding the progression of cervical lesions.

\section{Acknowledgements}

This study was supported by a grant from the National R\&D Program for Cancer Control, Ministry for Health, Welfare and Family affairs, Republic of Korea (grant no. 0820330).

\section{References}

1. Green J, Berrington de Gonzalez A, Sweetland S, et al: Risk factors for adenocarcinoma and squamous cell carcinoma of the cervix in women aged 20-44 years: the UK National Case-Control Study of Cervical Cancer. Br J Cancer 89: 2078-2086, 2003. 
2. Pisani P, Bray F and Parkin DM: Estimates of the world-wide prevalence of cancer for 25 sites in the adult population. Int $\mathrm{J}$ Cancer 97: 72-81, 2002.

3. Termini L, Maciag PC, Soares FA, et al: Analysis of human kallikrein 7 expression as a potential biomarker in cervical neoplasia. Int J Cancer 127: 485-490, 2010.

4. Lockwood WW, Coe BP, Williams AC, MacAulay C and Lam WL: Whole genome tiling path array $\mathrm{CGH}$ analysis of segmental copy number alterations in cervical cancer cell lines. Int J Cancer 120: 436-443, 2007.

5. Heselmeyer K, Schrock E, du Manoir S, et al: Gain of chromosome $3 q$ defines the transition from severe dysplasia to invasive carcinoma of the uterine cervix. Proc Natl Acad Sci USA 93: 479-484, 1996

6. Rapp L and Chen JJ: The papillomavirus E6 proteins. Biochim Biophys Acta 1378: F1-19, 1998.

7. Hillemanns P, Wang X, Staehle S, Michels W and Dannecker C: Evaluation of different treatment modalities for vulvar intraepithelial neoplasia (VIN): $\mathrm{CO}(2)$ laser vaporization, photodynamic therapy, excision and vulvectomy. Gynecol Oncol 100: 271-275, 2006.

8. Tranbaloc P: [Natural history of precursor lesions of cervical cancer]. Gynecol Obstet Fertil 36: 650-655, 2008 (In French).

9. Wilting SM, Steenbergen RD, Tijssen M, et al: Chromosomal signatures of a subset of high-grade premalignant cervical lesions closely resemble invasive carcinomas. Cancer Res 69: 647-655, 2009.

10. Ueda Y, Enomoto T, Miyatake T, et al: Monoclonal expansion with integration of high-risk type human papillomaviruses is an initial step for cervical carcinogenesis: association of clonal status and human papillomavirus infection with clinical outcome in cervical intraepithelial neoplasia. Lab Invest 83: 1517-1527, 2003.

11. van Zeeburg HJ, Snijders PJ, Pals G, et al: Generation and molecular characterization of head and neck squamous cell lines of fanconi anemia patients. Cancer Res 65: 1271-1276, 2005.

12. van der Laan MJ, Dudoit S and Pollard KS: Augmentation procedures for control of the generalized family-wise error rate and tail probabilities for the proportion of false positives. Stat Appl Genet Mol Biol 3: Jun 15, 2004 (Epub ahead of print).

13. Fitzpatrick MA, Funk MC, Gius D, et al: Identification of chromosomal alterations important in the development of cervical intraepithelial neoplasia and invasive carcinoma using alignment of DNA microarray data. Gynecol Oncol 103: 458-462, 2006.

14. Zhai Y, Kuick R, Nan B, et al: Gene expression analysis of preinvasive and invasive cervical squamous cell carcinomas identifies HOXC10 as a key mediator of invasion. Cancer Res 67: 10163-10172, 2007.

15. Alazawi W, Pett M, Strauss S, et al: Genomic imbalances in 70 snap-frozen cervical squamous intraepithelial lesions: associations with lesion grade, state of the HPV16 E2 gene and clinical outcome. Br J Cancer 91: 2063-2070, 2004.

16. Kang JU, Koo SH, Kwon KC, Park JW and Kim JM: Identification of novel candidate target genes, including EPHB3, MASP1 and SST at 3q26.2-q29 in squamous cell carcinoma of the lung. BMC Cancer 9: 237, 2009.

17. Milde T, Oehme I, Korshunov A, et al: HDAC5 and HDAC9 in medulloblastoma: novel markers for risk stratification and role in tumor cell growth. Clin Cancer Res 16: 3240-3252, 2010.

18. Yang Q, Jie Z, Cao H, et al: Low-level expression of let-7a in gastric cancer and its involvement in tumorigenesis by targeting RAB40C. Carcinogenesis 32: 713-722, 2011.

19. Matsuura M, Onimaru M, Yonemitsu Y, et al: Autocrine loop between vascular endothelial growth factor (VEGF)-C and VEGF receptor-3 positively regulates tumor-associated lymphangiogenesis in oral squamoid cancer cells. Am J Pathol 175: 1709-1721, 2009.

20. Habuchi H, Miyake G, Nogami K, et al: Biosynthesis of heparan sulphate with diverse structures and functions: two alternatively spliced forms of human heparan sulphate 6-O-sulphotransferase-2 having different expression patterns and properties. Biochem $\mathrm{J}$ 371: 131-142, 2003.

21. Narayan G, Pulido HA, Koul S, et al: Genetic analysis identifies putative tumor suppressor sites at 2q35-q36.1 and 2q36.3-q37.1 involved in cervical cancer progression. Oncogene 22: 3489-3499, 2003.
22. Rao PH, Arias-Pulido H, Lu XY, et al: Chromosomal amplifications, 3q gain and deletions of 2q33-q37 are the frequent genetic changes in cervical carcinoma. BMC Cancer 4: 5, 2004.

23. Zimmerman ES, Chen J, Andersen JL, et al: Human immunodeficiency virus type $1 \mathrm{Vpr}$-mediated $\mathrm{G} 2$ arrest requires Rad17 and Husl and induces nuclear BRCA1 and gamma-H2AX focus formation. Mol Cell Biol 24: 9286-9294, 2004.

24. Liang M, Ueno M, Oomizu S, et al: Galectin-9 expression links to malignant potential of cervical squamous cell carcinoma. J Cancer Res Clin Oncol 134: 899-907, 2008.

25. Tan GC, Sharifah NA, Shiran MS, Salwati S, Hatta AZ and Paul-Ng HO: Utility of Ki-67 and p53 in distinguishing cervical intraepithelial neoplasia 3 from squamous cell carcinoma of the cervix. Asian Pac J Cancer Prev 9: 781-784, 2008.

26. Tonk VS, Wilson GN, Yatsenko SA, et al: Molecular cytogenetic characterization of a familial der(1)del(1)(p36.33)dup(1) (p36.33p36.22) with variable phenotype. Am J Med Genet A 139A: 136-140, 2005.

27. Chen M, Ye Y, Yang H, et al: Genome-wide profiling of chromosomal alterations in renal cell carcinoma using high-density single nucleotide polymorphism arrays. Int J Cancer 125: 2342-2348, 2009.

28. Heilstedt HA, Ballif BC, Howard LA, Kashork CD and Shaffer LG: Population data suggest that deletions of $1 \mathrm{p} 36$ are a relatively common chromosome abnormality. Clin Genet 64: 310-316, 2003

29. Garnis C, Coe BP, Ishkanian A, Zhang L, Rosin MP and Lam WL: Novel regions of amplification on $8 \mathrm{q}$ distinct from the MYC locus and frequently altered in oral dysplasia and cancer. Genes Chromosomes Cancer 39: 93-98, 2004

30. Schlaepfer DD and Mitra SK: Multiple connections link FAK to cell motility and invasion. Curr Opin Genet Dev 14: 92-101, 2004.

31. Luo M,Fan H, Nagy T, et al: Mammary epithelial-specific ablation of the focal adhesion kinase suppresses mammary tumorigenesis by affecting mammary cancer stem/progenitor cells. Cancer Res 69: 466-474, 2009.

32. Ohkuni T, Kojima T, Ogasawara N, et al: Expression and localization of tricellulin in human nasal epithelial cells in vivo and in vitro. Med Mol Morphol 42: 204-211, 2009.

33. Benedicto I, Molina-Jimenez F, Bartosch B, et al: The tight junction-associated protein occludin is required for a postbinding step in hepatitis C virus entry and infection. J Virol 83: 8012-8020, 2009.

34. Li W, Wang W, Si M, et al: The physical state of HPV16 infection and its clinical significance in cancer precursor lesion and cervical carcinoma. J Cancer Res Clin Oncol 134: 1355-1361, 2008.

35. Singh RK, Dasgupta S, Bhattacharya N, et al: Deletion in chromosome 11 and Bcl-1/Cyclin D1 alterations are independently associated with the development of uterine cervical carcinoma. J Cancer Res Clin Oncol 131: 395-406, 2005.

36. Gallego MI, Schoenmakers EF, van de Ven WJ and Lazo PA: Complex genomic rearrangement within the 12q15 multiple aberration region induced by integrated human papillomavirus 18 in a cervical carcinoma cell line. Mol Carcinog 19: 114-121, 1997.

37. Lazo PA: The molecular genetics of cervical carcinoma. Br J Cancer 80: 2008-2018, 1999.

38. Reuter S, Bartelmann M, Vogt M, et al: APM-1, a novel human gene, identified by aberrant co-transcription with papillomavirus oncogenes in a cervical carcinoma cell line, encodes a BTB/ POZ-zinc finger protein with growth inhibitory activity. EMBO J 17: 215-222, 1998.

39. Thorland EC, Myers SL, Gostout BS and Smith DI: Common fragile sites are preferential targets for HPV16 integrations in cervical tumors. Oncogene 22: 1225-1237, 2003

40. Pollack JR, Sorlie T, Perou CM, et al: Microarray analysis reveals a major direct role of DNA copy number alteration in the transcriptional program of human breast tumors. Proc Natl Acad Sci USA 99: 12963-12968, 2002.

41. Hyman E, Kauraniemi P, Hautaniemi S, et al: Impact of DNA amplification on gene expression patterns in breast cancer. Cancer Res 62: 6240-6245, 2002 . 